\title{
Controllers design based on Takagi-Sugeno fuzzy systems and sliding mode for a non-holonomic mobile robot
}

\author{
HafedhAbid, \\ abidhafedh@gmail.com,
}

Laboratory of Sciences and Techniques of Automatic control \& computer engineering (Lab-STA) Sfax, Tunisia,

National School of Engineering of Sfax, University of Sfax, Tunisia

\begin{abstract}
:
This paper is interesting to a nonholonomic wheeled mobile robot. We have presented a scheme to develop controllers. Two controllers have been developed. The first concerns the kinematic behavior while the second relates to the dynamic behavior of the mobile robot. For the Kinematic controller, we have used a Takagi-Sugeno fuzzy system to overcome the nonlinearities present in model whereas for the second controller we have used the sliding mode approach. The sliding surface has the identical structure as the proportional integral controller. The stability of system has been proved based on Lyapunov approach. The simulation results show the efficiency of the proposed control laws.
\end{abstract}

Keywords: Nonholonomic mobile robot, Kinematic model, dynamic model, Lyapunov approach, , sliding mode controller, T-S fuzzy system.

\section{Introduction}

In the last decades, the path of travel is considered as one of the critical problems in the field of mobile robotics. The trajectory tracking consists of guiding the robot through intermediate points to reach the final destination. This tracking is done under a constraint time, which means that the robot must reach the goal within a predefined time. In the literature, the problem is treated as the tracking of a reference robot that moves to the desired trajectory with a certain rhythm. The real robot must follow precisely that of reference and reduce the distance error, by varying its linear and angular velocities [1], [2]. There are a many works that have focused on tracking the trajectory of the mobile robots, they consider the mobile robot as a particle, in this case the inputs are velocities. Their aims are kinematic models. In [10], the kinematic control law approach supposes that the control signal generates the exact motion commanded. On the other hand, some works consider the kinematic aspect and the dynamic aspect for the mobile robot. In this case, the actuator inputs signals are torques instead of velocities [3]. In [5], Jun-Ku Lee et al. suggest a technique for designing the tracking control of wheeled mobile robots based on a new sliding surface with an approach angle. In [7], authors proposed a robust backstepping controller for uncertain kinematic model of the wheeled mobile robot based on a nonlinear disturbance observer in order to cope with model uncertainties and the external disturbances. Venelinov [9] proposed an adaptive fuzzy approach for kinematic controller. This method was able to decrease the effect of unmodeled disturbances. In [11], a dynamic Petri recurrent fuzzy neural network was proposed. In [4], the proposed controller combines nonlinear time varying feedback with an integral sliding mode controller. The latter is obtained by introducing an integral term in the switching manifold.

In [12], a robust adaptive mobile robot controller is presented using backstepping for kinematics and dynamics motions, the adaptive process was based on the neural network. In [8] a classical 
parallel distributed compensation (PDC) control law, based on Takagi-Sugeno fuzzy modeling, is proposed. The controller comprises sixteen rules in which the control gains have been calculated using LMI techniques. In [19] authors, present an adaptive controller with consideration of unknown model parameters.

In [20] authors Suggest a controller of a mobile robot in Cartesian coordinates with an approach angle based on the sliding mode. In [22] authors Combine hybrid backstepping kinematic control with the adaptive integral sliding mode kinetic control of the three-wheeled mobile robot.

Most of the works, deal with Nonholonomic Wheeled Mobile Robot, use for kinematic motion a classical controller arising from the backstepping method [2, 12, 19, 21, 22].

This paper includes two main contributions. First a new controller based on Takagi-Sugeno fuzzy systems for kinematic motion. This last uses three fuzzy rules. The second contribution consists in developing for the dynamic part a controller based on the sliding mode. The sliding surface, which is based on linear and angular velocities of the robot, has the similar structure as the proportional integral controller. The switching control term of the latter controller combines the two sliding surfaces.

This paper is organized as follows. The next section is devoted to the description of the kinematic and dynamic models of the two-wheeled mobile robot.

The third section that is reserved to the controllers design includes two subsections, the first is reserved to the development of the new T-S type fuzzy controller of the kinematic behavior whereas the second, is consecrated to the design of the dynamic motion controller using the sliding mode approach. The stability analysis is chekked in the both precedent subsection by the Lyapunov approach. Then, the fourth section is sacred to the presentation of the simulation results.

\section{Mobile Robot Modeling}

In this section, we are interested in the modeling of the robot, which is composed of two driving wheels and a drive shaft in the center as shown in figure 1. Indeed, the first subsection is reserved for kinematic modeling while the second subsection concerns dynamic modeling.

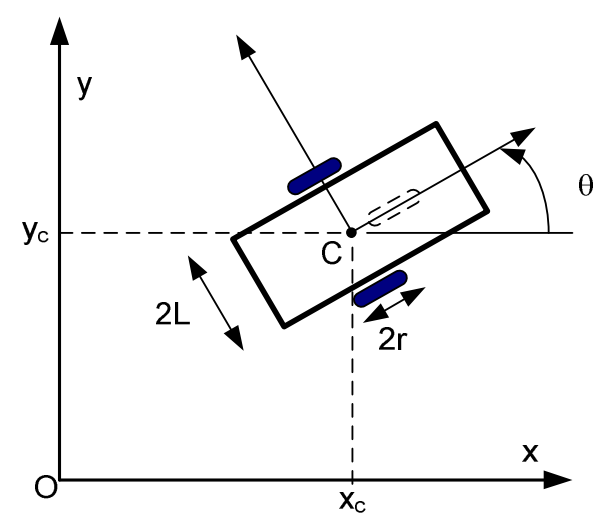

Fig 1: Representation of the Navigation Environment

We define the current position $\left(x_{c}, y_{c}\right)$ and the heading angle $\theta$, which constitutes the coordinates of middle point of the mobile robot and the angle between the heading direction and the $\mathrm{x}$-axis, to describe the current posture position of the mobile robot. The Fig. 1 depicts the current posture position of a two wheels mobile robot in Cartesian frame coordinates.

The nonholonomic constraint of a wheeled mobile robot is given by the following equation:

$\dot{y} \cos \theta-\dot{x} \sin \theta=0$ 


\subsection{Kinematic model}

Based on the Newton-Euler equations [13] and the previous hypothese, the state equations of mobile robot are represented by the following equations system [6]:

$$
\begin{aligned}
& \left\{\begin{array}{l}
\dot{x}=v \cos \theta \\
\dot{y}=v \sin \theta \\
\dot{\theta}=\omega
\end{array}\right. \\
& v=\sqrt{\dot{x}^{2}+\dot{y}^{2}}
\end{aligned}
$$

where $(x, y), v$ and $\omega$ represent respectively, the instantaneous position coordinates of point $\mathrm{C}$ of the mobile robot in the global Cartesian frame and the measurements at point $\mathrm{C}$ of the linear and angular speeds of the robot. The state variables of mobile robot are: $q=\left[\begin{array}{lll}x & y & \theta\end{array}\right]^{T}$.

$$
\begin{aligned}
& v_{d}=\sqrt{\dot{x}_{d}^{2}+\dot{y}_{d}^{2}} \\
& w_{d}=\frac{\dot{x}_{d} \ddot{y}_{d}-\dot{y}_{d} \ddot{x}_{d}}{\dot{x}_{d}^{2}+\dot{y}_{d}^{2}}
\end{aligned}
$$

where $v_{d}$ and $w_{d}$ represent respectively, the desired linear and angular velocity.

The state kinematic model of mobile robot in Cartesian frame coordinates is given by the following expression:

$$
\dot{q}=\left[\begin{array}{c}
\dot{x} \\
\dot{y} \\
\dot{\theta}
\end{array}\right]=\left[\begin{array}{cc}
\cos \theta & 0 \\
\sin \theta & 0 \\
0 & 1
\end{array}\right]\left[\begin{array}{c}
v(t) \\
w(t)
\end{array}\right]=J(\theta) V_{m}
$$

with, $V_{m}=\left[\begin{array}{ll}v & \omega\end{array}\right]^{T}, J(\theta)=\left[\begin{array}{ccc}\cos \theta & \sin \theta & 0 \\ 0 & 0 & 1\end{array}\right]^{T}$

\subsection{Dynamic model}

The dynamic equation of the wheeled mobile robot is given by the following equation:

$$
M(q) \ddot{q}+C(q, \dot{q}) \dot{q}+F(\dot{q})=B(q) \tau-A^{T}(q) \lambda
$$

where, $C(q, \dot{q})$ is the centripetal and Coriolis matrix, $F(\dot{q})$ is the friction force, $\tau$ represents the torque vector, $A^{T}(q)=0$,

$$
M(q)=\left[\begin{array}{ccc}
m & 0 & 0 \\
0 & m & 0 \\
0 & 0 & J_{g}
\end{array}\right] B(q)=\frac{1}{r}\left[\begin{array}{cc}
\cos \theta & \cos \theta \\
\sin \theta & \sin \theta \\
L & -L
\end{array}\right] C(q, \dot{q})=\left[\begin{array}{ccc}
0 & 0 & 0 \\
0 & 0 & 0 \\
0 & 0 & 0
\end{array}\right]
$$

$m$ and $J_{g}$ represent respectively the mass and the moment inertia of the wheeled mobile robot. $r$ and $L$ represent respectively the wheel radius and the distance separating the two driving wheels. Without considering disturbances and uncertainties, the latest equation becomes as:

$$
\bar{M}(q) \dot{V}_{m}=\bar{B}(q) \tau
$$

where, $\bar{M}(q)=\left[\begin{array}{cc}m & 0 \\ 0 & J_{g}\end{array}\right], \bar{B}(q)=\frac{1}{r}\left[\begin{array}{cc}1 & 1 \\ L & -L\end{array}\right], V_{m}=\left[\begin{array}{c}v \\ w\end{array}\right], \tau=\left[\begin{array}{c}\tau_{r} \\ \tau_{l}\end{array}\right]$

The expressions of linear and angular velocities of the mobile robot, $(v, w)$, depend on the left and right linear velocities of the motors. They are expressed by the following equations: 
$v=\frac{v_{r}+v_{l}}{2}$

$w=\frac{v_{r}-v_{l}}{2 L}$

\section{Design of Robot controllers}

In this work, we consider the kinematic and dynamic behavior of the robot. The purpose of the control design is to allow the robot to follow the virtual robot. The latter represents the reference robot and provides the desired path defined by the following vector: $q_{d}(t)=\left[\begin{array}{lll}x_{d} & y & \theta_{d}\end{array}\right]^{T}$

Therefore, the linear and the angular reference velocities $v_{d}$ and $\omega_{d}$ can be generated by regularly continuous control inputs.

$$
\dot{q}_{d}(t)=J\left(\theta_{d}\right) V_{m d}
$$

Where, $V_{m d}=\left[\begin{array}{ll}v_{d} & \omega_{d}\end{array}\right]^{T}$

The posture vector error is not specified in the global frame coordinate system, but quite as a vector error in the local frame coordinate system of the robot: $q_{e}(t)=\left[\begin{array}{lll}e_{1} & e_{2} & e_{3}\end{array}\right]^{T}$.

The posture vector error $q_{e}$ is computed basing on the actual posture vector $q(t)=\left[\begin{array}{lll}x & y & \theta\end{array}\right]^{T}$ and the reference posture vector $q_{d}(t)=\left[\begin{array}{lll}x_{d} & y_{d} & \theta_{d}\end{array}\right]^{T}$.

$\tilde{q}=q_{d}-q=\left[\begin{array}{l}x_{d}-x \\ y_{d}-y \\ \theta_{d}-\theta\end{array}\right]=\left[\begin{array}{l}e_{x} \\ e_{y} \\ e_{\theta}\end{array}\right]$

The relation between local frame and global frame, as shown in figure 2, is given by the following equation:

$q_{e}=\Re_{e} \tilde{q}$

Where, $\Re_{e}=\left[\begin{array}{ccc}\cos \theta & \sin \theta & 0 \\ -\sin \theta & \cos \theta & 0 \\ 0 & 0 & 1\end{array}\right]$

The equation (9) allows transforming the magnitudes described in the global coordinate system to the local coordinate system.

$\dot{q}_{e}=\dot{\mathfrak{R}}_{e} \tilde{q}+\mathfrak{R}_{e} \dot{\tilde{q}}$

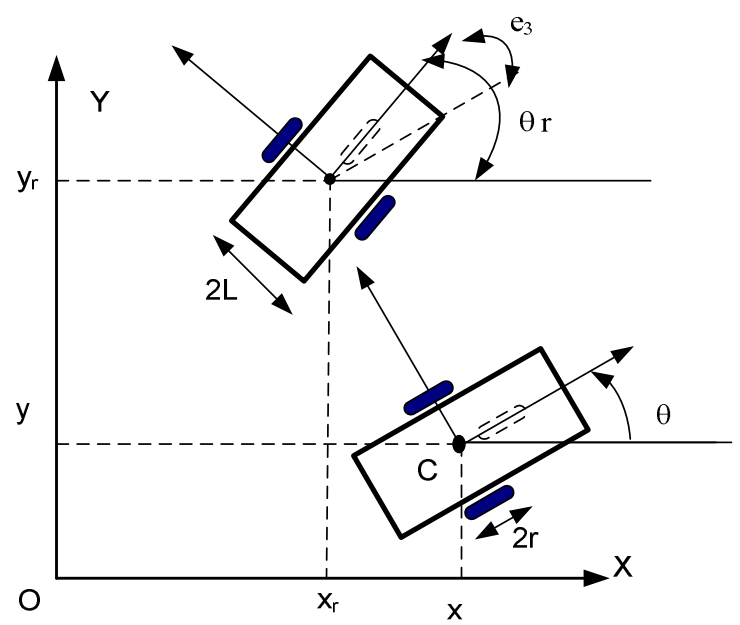


The architecture, of the control scheme of the robot, includes six blocks, as shown in Figure 3. The first block generates the desired states whereas the second block transforms the error from the local frame into the general frame. The third and fourth blocks are reserved respectively for kinetic and dynamic controllers. The fifth and sixth blocks respectively describe the behavior of the kinematic and dynamic models of the robot.

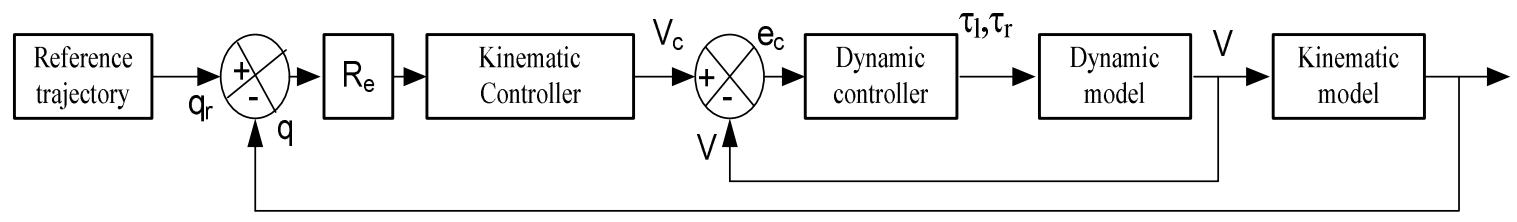

Fig 3: Architecture of robot Controller

\subsection{Fuzzy Kinematic controller}

In this subsection, we are interested in the search for a controller, which guarantees the convergence of the kinematic errors towards zero in the local coordinate system. However, by differentiating equation (13), which contains the linear speed and the angular speed terms, we obtain the derivative of the error vector, which is expressed by the following equation:

$$
\dot{q}_{e}=\left\{\begin{array}{l}
\dot{e}_{1}=\omega e_{2}-v+v_{d} \cos e_{3} \\
\dot{e}_{2}=-\omega e_{1}+v_{d} \sin e_{3} \\
\dot{e}_{3}=\omega_{d}-\omega
\end{array}\right.
$$

The posture error model can be rewritten as follow:

$$
\left[\begin{array}{l}
\dot{e}_{1} \\
\dot{e}_{2} \\
\dot{e}_{3}
\end{array}\right]=\left[\begin{array}{cc}
\cos e_{3} & 0 \\
\sin e_{3} & 0 \\
0 & 1
\end{array}\right]\left[\begin{array}{c}
v_{d} \\
w_{d}
\end{array}\right]+\left[\begin{array}{cc}
-1 & e_{2} \\
0 & -e_{1} \\
0 & 1
\end{array}\right]\left[\begin{array}{c}
v \\
w
\end{array}\right]
$$

Our goal is to find a control law that stabilizes the system and allows the robot to follow the desired path. For this reason, we use the fuzzy system. The advantage of the T-S type fuzzy approach is that it allows describing the nonlinear model by linear sub-models. Indeed, each sub-model represents a local linear relation between the inputs and the outputs and all the nonlinearities are reported in the premises of the fuzzy rules (Morère, 2001).

We note that equation (16) contains trigonometric nonlinearities which are cos (e3) and sin (e3). However, the nonlinearities depend on the error e3, whose range of variation is -pi / 2 to pi / 2. Based on the theory of T-S fuzzy systems, the nonlinear model (16) can be transformed into three local models, which are inferred by fuzzy rules. The three local models are described by the following systems of equations:

From the weights assigned to each rule, the state vector of the fuzzy models is inferred as follows (which corresponds to a barycentric aggregation).

The member ship function for the error $\mathrm{e}_{3}$ is given by figure 4 . 


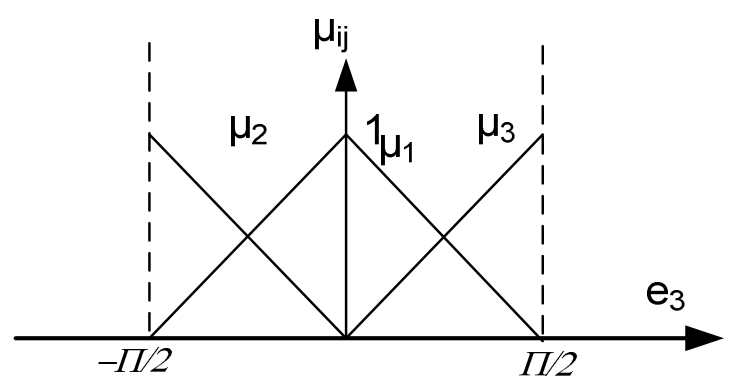

Fig 4Member ship function

The rules of the local models are given by the following expression

if $e_{3}$ is $\mu_{1}$ then $\dot{q}_{e}=A_{1} V_{m d}+B_{1} V_{m} \quad V=\left[v=K_{1} e_{1}+v_{d} \quad \omega=K_{3} e_{3}+v_{d} e_{2}+\omega_{d}\right]^{T}$

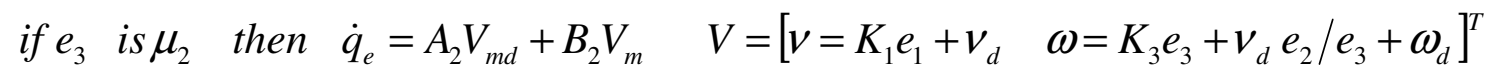

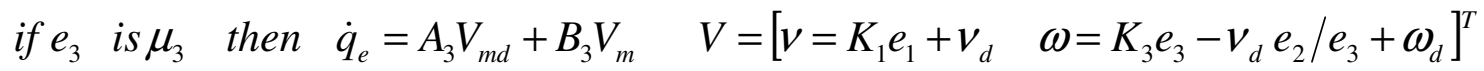

The T-S fuzzy model of equation (16) is given by the following equation:

$\dot{q}_{e}=\sum_{i=1}^{3} \mu_{i}\left(A_{i} V_{m d}+B_{i} V_{m}\right)$

Where, $\mu_{i}, A_{i}$ and $B_{i}$ represent respectively the weight assigned to each rule, the matrices associated to the local model.

The global control laws are:

$V_{m}=\left[v_{c}=\sum_{i=1}^{3} \mu_{i} v_{i}=K_{1} e_{1}+\mu_{1} v_{d} \quad \omega_{c}=\sum_{i=1}^{3} \mu_{i} \omega_{i}=\omega_{d}+K_{3} e_{3}+\mu_{1} v_{d} e_{2}+\left(\mu_{2}-\mu_{3}\right) v_{d} e_{2} / e_{3}\right]^{T}$

If $e_{3}=0$ then $\mu_{1}=1$ and $\mu_{2}=\mu_{3}=0$, so,

$V_{m}=\left[v_{c}=\sum_{i=1}^{3} \mu_{i} v_{i}=K_{1} e_{1}+\mu_{1} v_{d} \quad \omega_{c}=\sum_{i=1}^{3} \mu_{i} \omega_{i}=\omega_{d}+K_{3} e_{3}+\mu_{1} v_{d} e_{2}\right]^{T}$

\section{Stability analysis}

To check the stability of the robot, we use Lyapunov's theory. However, we choose the following Lyapunov candidate function.

$V=\frac{1}{2} e_{1}^{2}+\frac{1}{2} e_{2}^{2}+\frac{1}{2} e_{3}^{2}$

The derivative of Luapunov function is :

So, $\dot{V}=\dot{e}_{1} e_{1}+\dot{e}_{2} e_{2}+\dot{e}_{3} e_{3}$

$\dot{V}=\left(\mu_{1}\left(\omega e_{2}-v+v_{d}\right)+\mu_{2}\left(\omega e_{2}-v\right)+\mu_{3}\left(\omega e_{2}-v\right)\right) e_{1}+\left(\mu_{1}\left(-\omega e_{1}+v_{d} e_{3}\right)+\mu_{2}\left(-\omega e_{1}+v_{d}\right)+\mu_{3}\left(-\omega e_{1}-v_{d}\right)\right) e_{2}+$ $\left(\mu_{1}\left(\omega_{d}-\omega\right)+\mu_{2}\left(\omega_{d}-\omega\right)+\mu_{3}\left(\omega_{d}-\omega\right)\right) e_{3}$

So, $\dot{V}=\left(\left(-v+\mu_{1} v_{d}\right)\right) e_{1}+\left(\mu_{1} v_{d} e_{2}+\omega_{d}+\left(\mu_{2}-\mu_{3}\right) v_{d} e_{2} / e_{3}-\omega\right) e_{3}$

If we choose the following linear and angular velocities: 
$v_{c}=K_{1} e_{1}+\mu_{1} v_{d}, \omega_{c}=\sum_{i=1}^{3} \mu_{i} \omega_{i}=\omega_{d}+K_{3} e_{3}+\mu_{1} v_{d} e_{2}+\left(\mu_{2}-\mu_{3}\right) v_{d} e_{2} / e_{3}$

If $e_{3}=0 \quad$ then $\mu_{1}=1$ and $\mu_{2}=\mu_{3}=0$, so, $\omega_{c}=\sum_{i=1}^{3} \mu_{i} \omega_{i}=\omega_{d}+K_{3} e_{3}+\mu_{1} v_{d} e_{2}$

The equation (23) becomes as:

$\dot{V}=-K_{1} e_{1}^{2}-K_{3} e_{3}^{2} \leq 0$

The derivative of the Lyapunov function is negative and the stability of the system is guarantee.

\subsection{Dynamic controller based on sliding mode}

In this section, we are interested in the development of a controller, which guarantees the convergence of the posture error qe towards zero for any arbitrary reference trajectory. However, we have developed a controller based on the sliding mode approach because the latter is considered a robust approach. In this case, we define two sliding surfaces. The first surface depends on linear velocity while the second uses angular velocity, $S=\left[\begin{array}{ll}S_{v} & S_{w}\end{array}\right]^{T}$.

$S(t)=\left[\begin{array}{l}S_{v} \\ S_{w}\end{array}\right]=e_{e}(t)+K \int_{0}^{t} e_{e}(\delta) d \delta$

Where $s_{\mathrm{v}}$ and $s_{\mathrm{w}}$ are given respectively by equations (28) and (29), $e_{e}=\left(V_{c}-V_{m}\right)=\left[\begin{array}{ll}e_{v} & e_{w}\end{array}\right]^{T}$ With, $e_{v}=v_{c}-v$ and $e_{w}=w_{c}-w$.

$S_{v}(t)=e_{v}(t)+k_{v} \int_{0}^{t} e_{v}(\delta) d \delta$

$S_{w}(t)=e_{w v}(t)+k_{w} \int_{0}^{t} e_{w}(\delta) d \delta$

However, the derivatives of the sliding surfaces $s_{v}(t)$ and $s_{w}(t)$ are given by the following expressions:

$$
\begin{aligned}
& \dot{s}_{v}(t)=\dot{e}_{v}(t)+k_{v} e_{v}(t) \\
& \dot{s}_{w}(t)=\dot{e}_{w}(t)+k_{w} e_{w}(t)
\end{aligned}
$$

The dynamic motion of robot is described by equation (8) which can be transformed as:

$$
\dot{V}_{m}=(\bar{M}(q))^{-1} \bar{B}(q) \tau
$$

The equation (32) can be written as :

$\dot{V}_{m}=\tilde{B} \tau$

Where, $\tilde{B}=(\bar{M})^{-1} \bar{B}$

Basis on the sliding mode theory, the controller includes two terms which are known as equivalent control law and switching control. The global control law is expressed as:.

$u=\tau=u_{e q}+u_{s}=\tau_{e q}+\tau_{s}$

The equivalent control law $u_{e q}$ is computed $=0$ by recognizing that $\dot{S}=0$ which is a necessary condition for the state trajectory to stay in the sliding surface [17], [18]. The derivative of the sliding surface is:

$\dot{S}(t)=\dot{e}_{e}(t)+K e_{e}(t)$

With, $\dot{e}_{e}=\left(\dot{V}_{c}-\dot{V}_{m}\right)$ and $e_{e}=\left(V_{c}-V_{m}\right)$. 
Thus substituting (33) for (35), we obtain:

$\dot{S}(t)=\dot{V}_{c}-\tilde{B} \tau+K e_{e}(t)=\left[\begin{array}{l}0 \\ 0\end{array}\right]$

With, $\quad \bar{M}^{-1}=\frac{1}{m J_{g}}\left[\begin{array}{cc}J_{g} & 0 \\ 0 & m\end{array}\right], \widetilde{B}=\bar{M}^{-1} \bar{B}=\frac{1}{r m J_{g}}\left[\begin{array}{cc}J_{g} & J_{g} \\ m L & m L\end{array}\right]$,

$\widetilde{B}^{-1}=\frac{r}{2 L}\left[\begin{array}{cc}m L & J_{g} \\ m L & -J_{g}\end{array}\right], \bar{B}^{-1}=\frac{1}{2 L r}\left[\begin{array}{cc}L & 1 \\ L & -1\end{array}\right]$

However, the equivalent control law $u_{e q}=\tau_{e q}$ can be computed as:

$u_{e q}=\tau_{e q}=\widetilde{B}^{-1} \dot{V}_{c}+\widetilde{B}^{-1} K e_{e}(t)$

With,

$$
\dot{\mathrm{V}}_{\mathrm{c}}=\left[\begin{array}{c}
\dot{v}_{c}=K_{1} \dot{e}_{1}+\mu_{1} \dot{v}_{d} \\
\dot{\omega}_{c}=\dot{\omega}_{d}+K_{3} \dot{e}_{3}+\mu_{1}\left(\dot{v}_{d} e_{2}+v_{d} \dot{e}_{2}\right)+\left(\mu_{2}-\mu_{3}\right)\left(\left(\left(\dot{v}_{d} e_{2}+v_{d} \dot{e}_{2}\right) e_{3}-v_{d} e_{2} \dot{e}_{3}\right) / e_{3}^{2}\right)
\end{array}\right]
$$

So,

$\left[\begin{array}{c}\mathrm{u}_{\mathrm{eqr}} \\ \mathrm{u}_{\mathrm{eql}}\end{array}\right]=\left[\begin{array}{cc}\frac{r m}{2} & \frac{r J_{g}}{2 L} \\ \frac{r m}{2} & -\frac{r J_{g}}{2 L}\end{array}\right]\left(\left[\begin{array}{c}K_{1} \dot{e}_{1}+\mu_{1} \dot{v}_{d} \\ \dot{\omega}_{d}+K_{3} \dot{e}_{3}+\mu_{1}\left(\dot{v}_{d} e_{2}+v_{d} \dot{e}_{2}\right)+\left(\mu_{2}-\mu_{3}\right)\left(\left(\dot{v}_{d} e_{2}+v_{d} \dot{e}_{2}\right) e_{3}-v_{d} e_{2} \dot{e}_{3}\right) / e_{3}\end{array}\right]+\left[\begin{array}{l}\lambda e_{v} \\ \lambda e_{w}\end{array}\right]\right)$

$\left[\begin{array}{c}\mathrm{u}_{\mathrm{eqr}} \\ \mathrm{u}_{\mathrm{eql}}\end{array}\right]=\left[\begin{array}{cc}\frac{r m}{2} & \frac{r J_{g}}{2 L} \\ \frac{r m}{2} & -\frac{r J_{g}}{2 L}\end{array}\right]\left[\begin{array}{c}K_{1} \dot{e}_{1}+\mu_{1} \dot{v}_{d}+\lambda e_{v} \\ \dot{\omega}_{d}+K_{3} \dot{e}_{3}+\mu_{1}\left(\dot{v}_{d} e_{2}+v_{d} \dot{e}_{2}\right)+\left(\mu_{2}-\mu_{3}\right)\left(\dot{v}_{d} e_{2} / e_{3}+v_{d} \dot{e}_{2} / e_{3}-v_{d} e_{2} \dot{e}_{3} / e_{3}^{2}\right)+\lambda e_{w}\end{array}\right]$

$\left[\begin{array}{l}\mathrm{u}_{\mathrm{eqr}} \\ \mathrm{u}_{\mathrm{eql}}\end{array}\right]=\left[\begin{array}{l}\frac{r m}{2}\left(K_{1} \dot{e}_{1}+\mu_{1} \dot{v}_{d}+\lambda e_{v}\right)+\frac{r J_{g}}{2 L}\left(\dot{\omega}_{d}+K_{3} \dot{e}_{3}+\mu_{1}\left(\dot{v}_{d} e_{2}+v_{d} \dot{e}_{2}\right)+\left(\mu_{2}-\mu_{3}\right)\left(\frac{\left(\dot{v}_{d} e_{2}+v_{d} \dot{e}_{2}\right) e_{3}-v_{d} e_{2} \dot{e}_{3}}{e_{3}^{2}}\right)+\lambda e_{w}\right) \\ \frac{r m}{2}\left(\left(K_{1} \dot{e}_{1}+\mu_{1} \dot{v}_{d}+\lambda e_{v}\right)\right)-\frac{r J_{g}}{2 L}\left(\dot{\omega}_{d}+K_{3} \dot{e}_{3}+\mu_{1}\left(\dot{v}_{d} e_{2}+v_{d} \dot{e}_{2}\right)+\left(\mu_{2}-\mu_{3}\right)\left(\frac{\left(\dot{v}_{d} e_{2}+v_{d} \dot{e}_{2}\right) e_{3}-v_{d} e_{2} \dot{e}_{3}}{e_{3}^{2}}\right)+\lambda e_{w}\right)\end{array}\right]$

Finally, we obtain,

$\mathrm{u}_{\mathrm{eqr}}=\frac{r m}{2}\left(K_{1} \dot{e}_{1}+\mu_{1} \dot{v}_{d}+\lambda e_{v}\right)+\frac{r J g}{2 L}\left(\dot{\omega}_{d}+K_{3} \dot{e}_{3}+\mu_{1}\left(\dot{v}_{d} e_{2}+v_{d} \dot{e}_{2}\right)+\left(\mu_{2}-\mu_{3}\right)\left(\frac{\left(\dot{v}_{d} e_{2}+v_{d} \dot{e}_{2}\right) e_{3}-v_{d} e_{2} \dot{e}_{3}}{e_{3}^{2}}\right)+\lambda e_{w}\right)$
$\mathrm{u}_{\mathrm{eql}}=\frac{r m}{2}\left(\left(K_{1} \dot{e}_{1}+\mu_{1} \dot{v}_{d}+\lambda e_{v}\right)\right)-\frac{r J g}{2 L}\left(\dot{\omega}_{d}+K_{3} \dot{e}_{3}+\mu_{1}\left(\dot{v}_{d} e_{2}+v_{d} \dot{e}_{2}\right)+\left(\mu_{2}-\mu_{3}\right)\left(\frac{\left(\dot{v}_{d} e_{2}+v_{d} \dot{e}_{2}\right) e_{3}-v_{d} e_{2} \dot{e}_{3}}{e_{3}^{2}}\right)+\lambda e_{w}\right)$

The switching control term is generally chooses as: $u_{s}=-\eta \operatorname{sign}(S)$, with $\eta \succ 0$. In this paper, the switching control law is chosen as follows: 
$\tau_{s}=\left[\begin{array}{l}-k_{s 1}\left(\operatorname{sgn}\left(s_{v}+s_{w}\right)\right) \\ -k_{s 1}\left(\operatorname{sgn}\left(s_{v}-s_{w}\right)\right)\end{array}\right]$

\section{Reaching condition and Stability Analysis}

To verify the reaching condition, we need to just check the following condition. The Lyapunov candidate function is choosing as:

$V=\frac{1}{2} S^{T} S$

The derivative can be expressed as :

$\dot{V}=S^{T} \dot{S}$

$\dot{V}=S^{T}\left[\dot{V}_{c}-\tilde{B} \tau+K e_{e}(t)\right]$

$\dot{V}=S^{T}\left[\dot{V}_{c}-\tilde{B}\left(\tau_{e q}+\tau_{s}\right)+K e_{e}(t)\right]$

$\dot{V}=S^{T} \dot{S}=S^{T}\left[\dot{V}_{c}-\tilde{B} \tau_{e q}+K e_{e}(t)-\tilde{B} \tau_{s}\right]$

Basis on the equation (37) $\dot{V}_{c}-\widetilde{B} \tau_{e q}+K e_{e}(t)=0$

So,

$\dot{V}=S^{T} \dot{S}=S^{T}\left[\begin{array}{cc}\frac{r m}{2} & \frac{r J_{g}}{2 L} \\ \frac{r m}{2} & -\frac{r J_{g}}{2 L}\end{array}\right]\left[\begin{array}{l}-k_{s}\left(\operatorname{sgn}\left(s_{v}+s_{w}\right)\right) \\ -k_{s}\left(\operatorname{sgn}\left(s_{v}-s_{w}\right)\right)\end{array}\right]$

$\dot{V}=S^{T}\left[\begin{array}{l}-k_{s} \frac{r m}{2} \operatorname{sgn}\left(s_{v}+s_{w}\right)-k_{s} \frac{r J_{g}}{2 L} \operatorname{sgn}\left(s_{v}-s_{w}\right) \\ -k_{s} \frac{r m}{2} \operatorname{sgn}\left(s_{v}+s_{w}\right)+k_{s} \frac{r J_{g}}{2 L} \operatorname{sgn}\left(s_{v}-s_{w}\right)\end{array}\right]$

$\dot{V}=\left[\begin{array}{ll}s_{v} & s_{w}\end{array}\right]\left[\begin{array}{l}-k_{s} \frac{r m}{2} \operatorname{sgn}\left(s_{v}+s_{w}\right)-k_{s} \frac{r J_{g}}{2 L} \operatorname{sgn}\left(s_{v}-s_{w}\right) \\ -k_{s} \frac{r m}{2} \operatorname{sgn}\left(s_{v}+s_{w}\right)+k_{s} \frac{r J_{g}}{2 L} \operatorname{sgn}\left(s_{v}-s_{w}\right)\end{array}\right]$

$\dot{V}=-\frac{k_{s} r m\left(s_{v}+s_{w}\right)}{2} \operatorname{sgn}\left(s_{v}+s_{w}\right)-\frac{k_{s} r J_{g}\left(s_{v}-s_{w}\right)}{2 L} \operatorname{sgn}\left(s_{v}-s_{w}\right)$

$\dot{V}=-\frac{k_{s} r m}{2}\left|\left(s_{v}+s_{w}\right)\right|-\frac{k_{s} r J_{g}}{2 L}\left|\left(s_{v}-s_{w}\right)\right|$

$\dot{V}=-k_{s}\left|s_{v}+s_{w}\right|-k_{s}\left|s_{v}-s_{w}\right| \prec 0$

\section{Simulation results}


In this part, we present the simulation results of the nonholonomic wheeled mobile robot. The parameters of the robot (see figure 1) are: $\mathrm{m}=17 \mathrm{~kg}, J=0.537 \mathrm{~kg} \cdot \mathrm{m}^{2}, L=0.24 \mathrm{~m}, r=0.1 \mathrm{~m}$ and initial position $\left[\begin{array}{lll}x_{o} & y_{o} & \theta\end{array}\right]=\left[\begin{array}{lll}5 & 1 & \pi / 12\end{array}\right]$. The reference speed has been chosen variable as shown by the following equation:

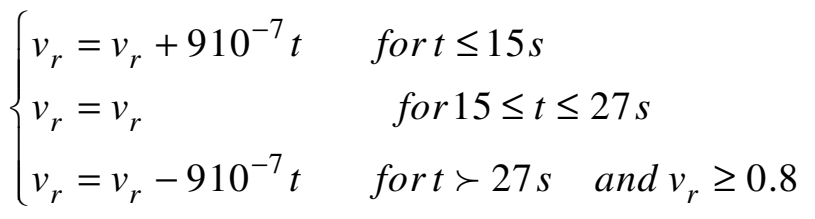

The initial speeds have been choose as $v_{d}=0.78, v_{g}=0.38$.

The following figures 5, 6 show respectively the evolution of the robot along the $\mathrm{x}$ and $\mathrm{y}$ axis (the trajectories and the reference)

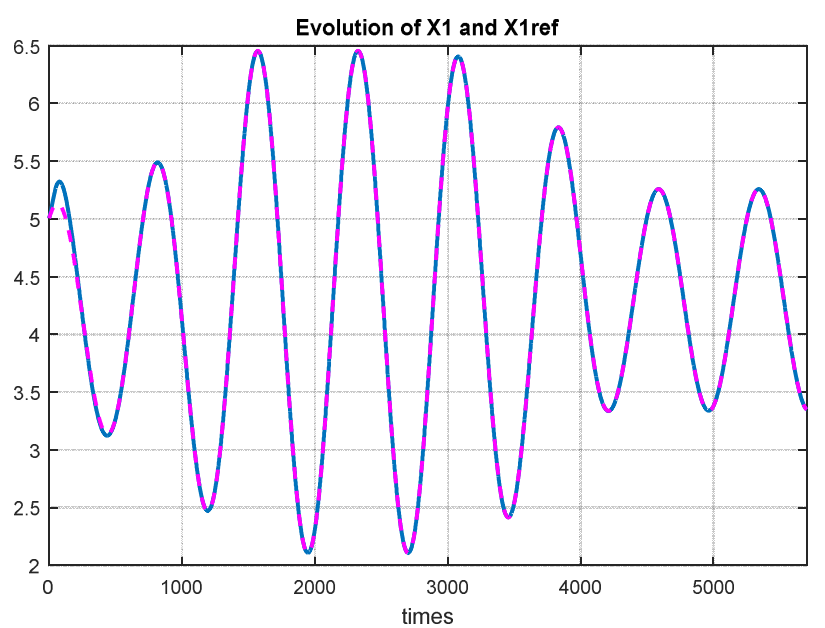

Figure 5: Evolution of the robot trajectory along the $\mathrm{x}$ axe (in blue the trajectory and in magenta the reference trajectory)

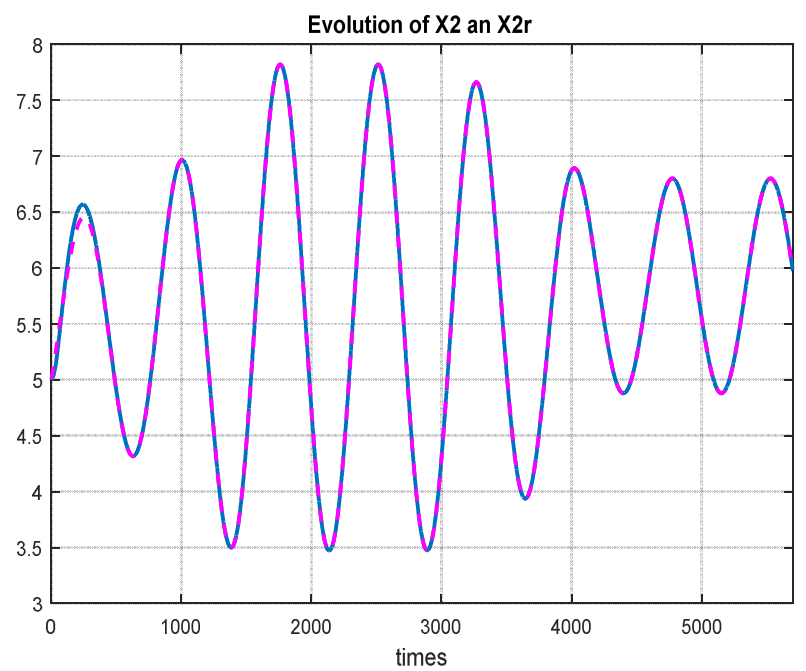

Figure 6: Evolution of the robot trajectory along the y axe (in blue the trajectory and in magenta the reference trajectory). 
Figure 7 shows the behavior evolution of linear speed v.

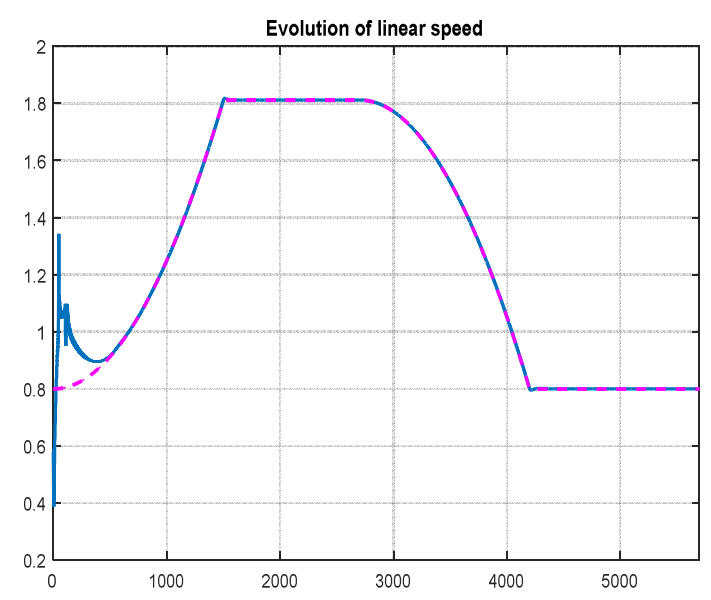

Figure 7: Evolution of linear speed v.

Figures 5, 6 and 7 show that all the signals follow quickly the reference one. The curves of the reference signals and the responses overlap in a very short time after start-up.

The figure 8 shows the evolution the angular error $\mathrm{e}_{3}$.

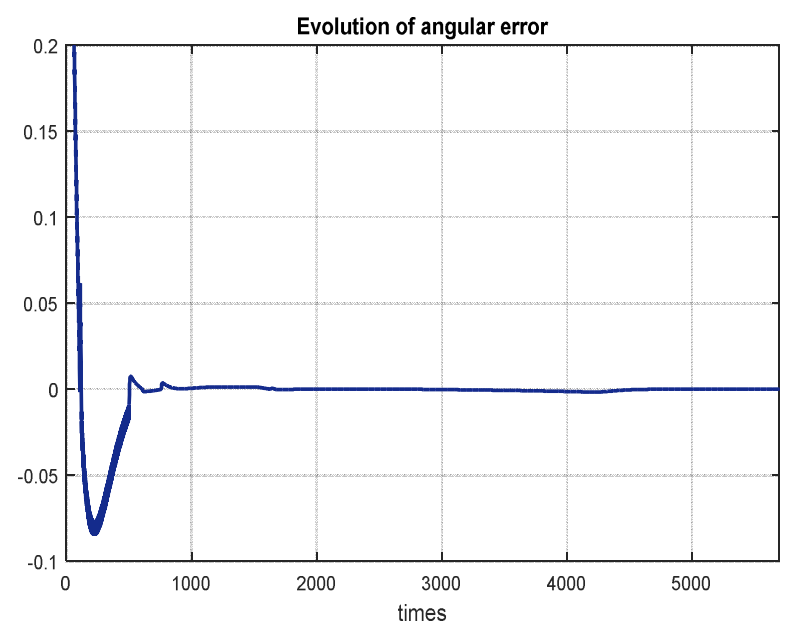

Figure 8: Evolution the angular error $\mathrm{e}_{3}$

Figures 9 and 10 show respectively the behavior evolution of errors $\mathrm{e}_{2}$ and $\mathrm{e}_{1}$. 


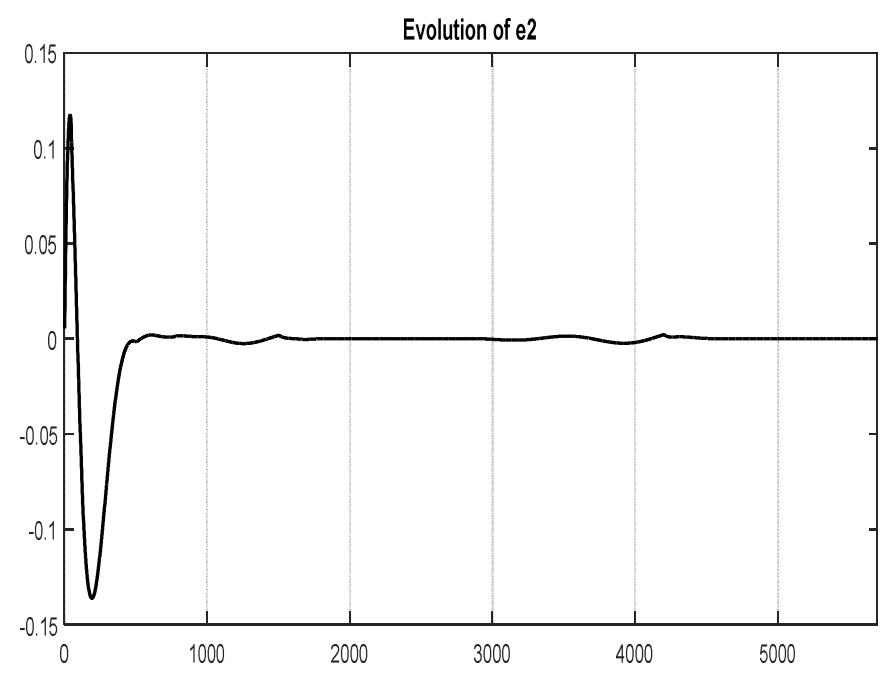

Figure 9: Evolution the angular error $\mathrm{e}_{2}$

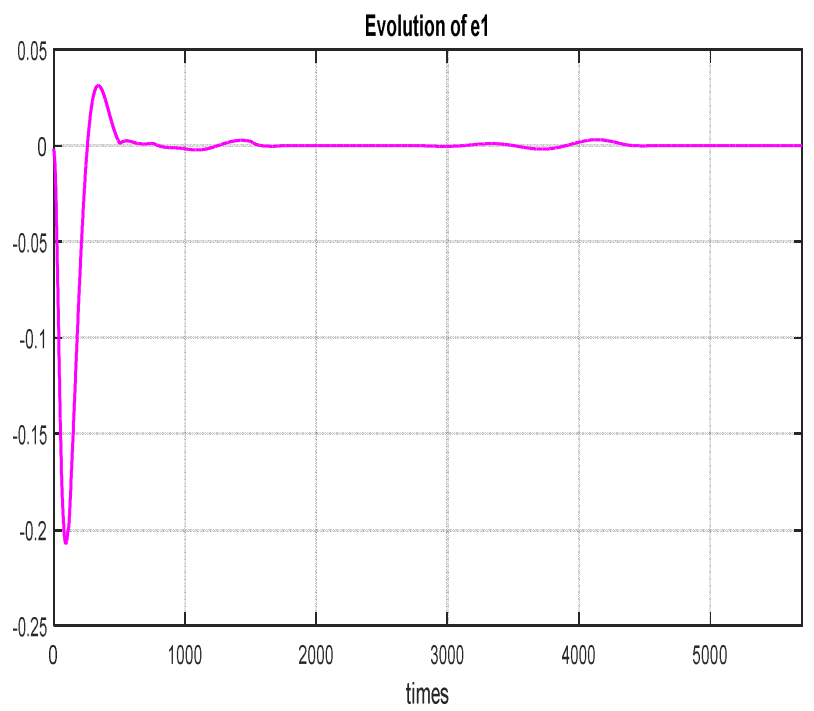

Figure 10: Evolution the angular error $\mathrm{e}_{1}$.

Figures 8,9 and 10: show that the errors $\mathrm{e}_{1}, \mathrm{e}_{2}$ and $\mathrm{e}_{3}$ rapidly tend towards zero. This clearly shows that the system converges towards the desired trajectories in a very short time. This proves the effectiveness of the proposed command.

\section{Conclusion}

In this paper, we have presented a scheme to control a nonholonomic robot. We have proposed two controllers. The first concerns the kinematic behavior while the second relates to the dynamic behavior of the mobile robot. To overcome the nonlinearities present in model we have used a Takagi-Sugeno fuzzy system for the Kinematic controller. For the second controller we have used the sliding mode technique, which is known as a robust technique. The proposed sliding surface has the same structure as the proportional integral controller. Lyapunov approach has been used to prove stability of system. The results presented in section 4 show the efficiency of the proposed control laws. 


\section{Conflict of Interest}

All Author, Hafedh ABID, declare that there is no conflict of interest.

\section{References}

[1]Takanori Fukao, Hiroshi Nakagawa, and Norihiko Adachi, "Adaptive Tracking Control of a Non- holonomic Mobile Robot", IEEE transactions on robotics and automation, vol.16, $\mathrm{n}^{\circ} 5$, October,pp609-615, 2000.

[2] Ghania Zidani, Said Drid, Larbi Chrifi-Alaoui, AbdeslamBenmakhlouf, Souad Chaouch, "Back- stepping Controller for a Wheeled Mobile Robot", 4thInternationalvConferenceon Systemsand Control (ICSC'15),April 28-30,2015, Sousse,Tunisia.

[3] J. Yang, J. kim, "Sliding mode motion control of nonholonomic mobile robots", IEEE control systems, Mag. 19 (2), pp 15-23, 1999.

[4] Aicha Bessas, Atallah Benalia, and Farès Boudjema, "Integral Sliding Mode Control for Trajectory Tracking of Wheeled Mobile Robot in Presence of Uncertainties",Hindawi Publishing Corporation Journal of Control Science and Engineering Volume 2016, Article ID 7915375, 10

[5] Jun-Ku Lee, Jin-Bae Park and Yoon-Ho Choi, "Tracking Control of Nonholonomic Wheeled Mobile Robot Based on New Sliding Surface with Approach Angle”, 3rd IFAC Symposium on Telematics Applications The International Federation of Automatic Control November 11-13, 2013, Seoul, Korea.

[6] M. K. Bugeja, S. Fabri, and L. G Camilleri, "Dual Adaptive Dynamic Control of Mobile Robots Using Neural Networks", IEEE Transactions on Systems, Man, and Cybernetics, Part B: Cybernetics,vol. 39, no. 1, pp. 129-141, 2009.

[7]Mahmood Ali MoqbelObaid, Abdul Rashid Husain and Ali Abdo Mohammed Al-kubati, "Robust Backstepping Tracking Control of Mobile Robot Based on Nonlinear Disturbance Observer", International Journal of Electrical and Computer Engineering (IJECE), Vol. 6, No. 2, April 2016, pp. 901-908.

[8] El-Hadi Guechi J Jimmy Lauber· Michel Dambrine · Gregor Klancar ` · Sašo Blažic, “ PDC Control Design for Non-holonomic Wheeled Mobile Robots with Delayed Outputs", Journal of Intelligent \& Robotic Systems December 2010 60(3):395-414

[9] A. VenelinovTopalov, J.-H.Kim and T. Proychev, "Fuzzy-Net Control of Non-Holonomic Mobile Robot Using Evolutionary Feedback-Error-Learning”, Robotics and Autonomous Systems, Vol. 23, No. 3, 1998, pp. 187-200.

[10] M. Egerstedt, X. Hu and A. Stotsky, "Control of Mobile Platforms Using a Virtual Vehicle Approach",IEEE Transactions on Automatic Control, Vol. 46, No. 11,2001, pp. 1777-1782.

[11] R.-J. Wai and C.-M. Liu, "Design of Dynamic Petri Re-current Fuzzy Neural Network and Its Application to Path-Tracking Control of Nonholonomic Mobile Robot", IEEE Transactions on Industrial Electronics, Vol. 56, No.7, 2009, pp. 2667-2683.

[12]R. Fierro and F. Lewis, "Control of a Nonholonomic Mo-bile Robot: Backstepping Kinematics into Dynamics", Journal of Robotic Systems vol 14 N 3, pp149-163 (1997)

[13] J. Craig, "Introduction to Robotics: Mechanics and Control", 1em plus $0.5 \mathrm{em}$ minus $0.4 \mathrm{em}$ Addison-Wesley, 1989.

[14] H Lee and M. Tomizuka, "Robuste adaptive control using a universal approximator for SISO nonlinear systems", IEEE transactions Fuzzy Systems, volume $8 \mathrm{~N}^{\circ} 1$, pp 95-106, February 2000. [15] Ying, H. "General SISO Takagi-Sugeno fuzzy systems with linear rule consequent are universal approximators", IEEE Transactions on Fuzzy Systems, Letters, November, Vol. 6, No. 4, pp.582-1587, 1998.

[16] Cao, S.G., Rees N.W. and Feng, G. "Universal fuzzy controllers for a class of non-linear 
systems", Fuzzy Sets and Systems, Vol. 122, pp.117-123, 2001.

[17] Hafedh Abid, Mohamed Chtourou and Ahmed Toumi, "An indirect adaptive fuzzy sliding mode controller for a class of SISO non-linear systems”, Int. J. Modelling, Identification and Control, Vol. 4, No. 2, 2008

[18] Utkin, "Variable structure systems with sliding mode", IEEE Transactions on Automatic Control, April, Vol. AC-22, No. 2, pp.212-222, 1997.

[19] Hung-Hsiu Yu, Hsuan-Kuan Huang, "Experimental Verification for Adaptive Tracking

Control of a Nonholonomic Mobile Robot", The 33rd Annual Conference of the IEEE Industrial Electronics Society (IECON) Nov. 5-8, 2007, Taipei, Taiwan

[20] Jun Ku Lee, Yoon Ho Choi, and Jin Bae Park, "Sliding Mode Tracking Control of Mobile Robots with Approach Angle in Cartesian Coordinates", International Journal of Control, Automation, and Systems (2015) 13(3):718-724 DOI 10.1007/s12555-014-0024-5

[21] Khadir Lakhdar Besseghieur1 , Radosław Trębiński1, Wojciech Kaczmarek1, Jarosław Panasiuk1, «Trajectory tracking control for a nonholonomic mobile robot under ROS", ICMCE IOP Publishing, IOP Conf. Series: Journal of Physics: Conf. Series 1016 (2018) 012008 [22] Abolfath Nikranjbar, Masoud Haidari a, Ali Asghar Atai b, "Adaptive Sliding Mode Tracking Control of Mobile Robot in Dynamic Environment Using Artificial Potential Fields », Journal of Computer \& Robotics 11 (1), 2018 1-14 